\title{
Enhanced N-Cut and Watershed based Model for Brain MRI Segmentation
}

\author{
Naresh Ghorpade, H. R. Bhapkar
}

\begin{abstract}
Segmentation of an image is most important and essential task in medical image processing, specifically while analyzing magnetic resonance (MR) image of brain clinically. during the clinical investigation of brain MRI images. Lot of research has been carried out for MRI segmentation but still it is challenging task. Hybrid approach which uses enhanced normalized cut and watershed transform to segment brain MRI images is developed in this paper. Watershed transform is used for the initial partitioning of the MRI, which creates primitive regions. In the next stage these primitive regions resembled for graph depiction and then the normalized cut method is used for segmenting an image. Variety of simulated and actual MR images are being segmented by using proposed algorithm to test its efficiency, in addition to it segmentation results are also compared with the other available techniques of brain MRI segmentation.

Keywords: Brain MRI Segmentation, Watershed Transform, Graph Partitioning, Normalized Cut.
\end{abstract}

\section{INTRODUCTION}

Dimensional analysis and variance among the soft tissues are the main important features of an advanced magnetic resonance imaging (MRI) technique [1]. The refinement and investigations of health descriptions plays vital role in the diagnosis. MRI is the most suitable option for the study of brain due to its capability of producing better contrast resolution [2]. Brain image analysis mainly focuses on the tissues like white matter (WM), grey matter (GM), and cerebral spinal fluid (CSF). To analyse an image and diagnose the disease variations in the tissue textures either in the whole image or into the specific sections are observed [3 4]. Partitioning of an image into nonoverlapping regions such that each region possess specific property is called as image segmentation. Brain MRI segmentation indicates assignment of the tissue type to every pixel of two dimensional and three-dimensional region by observing brain MRI images and the earlier history. It is the initial phase in numerous medical investigation and medical applications, viz. calculation of tissue dimensions, multimodal grouping and process, competent brain mapping. Noise, dissimilarity in the intensity and limited volume significance are the vital features disturbing to the MRI image segmentation. Lot of

Revised Manuscript Received on May 25, 2020.

* Correspondence Author

Naresh Ghorpade*, Department of Mathematics, MIT School of Engineering, MITADT University, Loni Kalbhor, Pune - 412201, Maharashtra, India; E-mail: nareshghorpade1@yahoo.co.in.

H. R. Bhapkar, Department of Mathematics, MIT School of Engineering, MITADT University, Loni Kalbhor, Pune - 412201, Maharashtra, India; E-mail: hrbhapkar@gmail.com.

(C) The Authors. Published by Blue Eyes Intelligence Engineering and Sciences Publication (BEIESP). This is an open access article under the CC BY-NC-ND license (http://creativecommons.org/licenses/by-nc-nd/4.0/) research has been carried out for segmentation of Brain MRI images. Most of the methods are based on three categories; classification, region and boundary [5]. Based on some specific measure every pixel gets assigned to the particular category of tissue in the classification methods. Thresholding [6], statistical grading [7] and grouping [8] are the methods of this category. Region growing approach [9] and watershed separation [10], focuses on identifying distinct similar regions with respect to various objects in an image. Boundary based segmentation technique also considers gradient features which are closed to the boundary of an object as a baseline. Detection of an edge [11], deformable prototypes [12] and vigorous curves [13] are boundary-based segmentation techniques. Classification and region-based methods have restrictions on performing well due to intensity differences, noise disturbances and counterfeit arcs affects the quality of segmentation in the methods based on boundary. Rather than these elementary segmentation methods, some additional approaches are also addressed in the literature. MRI segmentation based on artificial neural networks (ANNs) is used in [14] [15]. Fuzzy C-Means (FCM) [16] is the well-known method used for brain MRI segmentation. Watershed and graph partitioning based segmentation is proposed in [17]; Piecewise continuity is used in this method to partition the graph. In the literature several image brain MRI segmentation methods and their enhancements has been addressed but still certain areas in the brain image analysis are neglected and need to develop efficient brain MRI segmentation techniques.

Hybrid approaches based on various graph theoretical segmentation methods will be helpful to address the pitfalls in the existing methods.

We have proposed a novel hybrid approach based on enhanced normalize cut and watershed transform for segmentation of brain MRI. Organization of the paper is as below: Conventional watershed section and improved N-Cut technique is illustrated in section 2. Proposed Watershed Enhanced Normalized Cut Algorithm (WENCA) is developed in Section3. Performance of the proposed algorithm is evaluated and analyzed in Section 4 and Section 5 conclude the paper.

\section{WATERSHED TRANSFORM AND NORMALIZED CUTS}

\subsection{Watershed Transform}

Watershed transform is region-based segmentation technique which uses mathematical morphology.

Published By:

Blue Eyes Intelligence Engineering

Retrieval Number: E9535069520/2020@BEIESP

DOI: 10.35940/ijeat.E9535.069520

Journal Website: www.ijeat.org

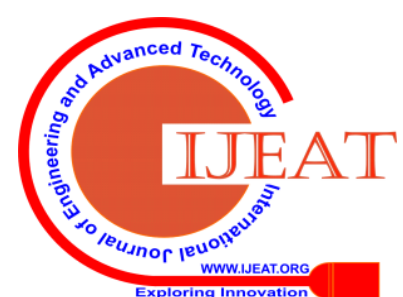




\section{Enhanced N-Cut and Watershed based Model for Brain MRI Segmentation}

Geologically, a watershed is the contour line separating the regions voided by distinct rivers. A catchment basin is an area draining into a river or basin. Same perception is used by the watershed transform for segmenting variety of images. For this approach topological surface is an image such that function values of each pixel indicates height, as result visualization of an image is three-dimensional surface. Catchment basin is an area where the water gets collected by the assumption that rain is falling on this surface. Rain falling precisely on the watershed edge contour would be correspondingly expected to collect in either of the two catchment basins. To identify the catchment basin and connect the contour of an image is the role of watershed transform, gradient magnitude is used for primary processing of an image. In the raw image object counters will have high pixel values whereas low pixel values are scattered all over in the remaining region of an image. Preferably, then, the watershed transform generates watershed boundaries through object curves. Gradient Watershed transform is used for the primary grouping of the MRI, which creates primitive regions.

\subsection{Normalized Cut Methods}

Vertex set of every graph $G=(P, Q)$ having more than one vertex can be divided into two disjoint sets $J$ and $K$. Summation of weights of edges among the sets $J$ and $K$ is cut value.

$\operatorname{Cut}(J, K)=\sum_{p a \in J, b \in K} w(a, b)$

Optimization of the cut value is main objective of the partitioning. To identify the minimum cut value all possible partitions need to be considered, which is very difficult task in case of graphs with maximum number of vertices and edges. Variety of methods has been proposed to optimize cut value while partitioning the graph. Clustering based on minimum cut is proposed by Wu et al. [18], but it works well only for the graphs with a smaller number of vertices. This drawback is being well addressed by Shi et al. [19], they have proposed normalized cut for partitioning. For a graph partition, $R=J \cup K$ the cost of normalized cut is

$$
\operatorname{Ncut}(J, K)=\frac{\operatorname{Cut}(J, K)}{\operatorname{assoc}(J, R)}+\frac{\operatorname{Cut}(J, K)}{\operatorname{assoc}(K, R)}
$$

where summation of weights of all the edges removed for dividing the graph is $\operatorname{Cut}(J, K)$ whereas $\operatorname{Assoc}(J, R)$ and $\operatorname{Assoc}(K, R)$ is the summation of edge weight connecting the vertices of $J$ to the vertices of original graph $G$ and edge weight connecting the vertices of $K$ to the vertices in the original graph $G$ respectively. Higher proportion of adjacencies among the set and remaining nodes leads to the smaller value of Ncut in the disassociation.

Likewise, entire normalized association inside the groups for specified partition is

$$
\operatorname{Ncut}(J, K)=\frac{\operatorname{assoc}(J, J)}{\operatorname{assoc}(J, R)}+\frac{\operatorname{assoc}(K, K)}{\operatorname{assoc}(K, R)}
$$

where summation of edge weight joining the vertices inside $J$ and $K$; are $\operatorname{assoc}(J, J)$ and $\operatorname{assoc}(K, K)$ respectively. This is an indicatives measure for strong connection among the vertices within the group.

Relation between measures of association and disassociation in the partitioning is:

$$
\text { Ncut }=2-\text { Nassoc }
$$

This relation indicates that the main criteria of partitioning to minimize the disassociation among the parts and association within the part can be fulfilled simultaneously.

\section{Ncut Optimization:}

Let $P$ vertex set off graph $G$ partitioned into two sets $L$ and $M$, then the minimal value of normalized cut for a graph with $V$ vertices is determined by using following steps:

- Let $d(a)=\sum w(a, b)$, sum of weight of all the edges between node $a$ to the other nodes in the graph G.

- Let $P=\left[\begin{array}{cccc}p_{1} & 0 & \cdots & 0 \\ 0 & p_{2} & \ldots & 0 \\ \vdots & \vdots & \ddots & \vdots \\ 0 & 0 & \ldots & p_{k}\end{array}\right]$

be diagonal matrix of degrees and

$$
Q=\left[\begin{array}{cccc}
q_{11} & q_{12} & \cdots & q_{1 k} \\
q_{21} & q_{22} & \cdots & d_{2 k} \\
\vdots & \vdots & \ddots & \vdots \\
q_{l 1} & q_{l 2} & \cdots & q_{l k}
\end{array}\right]
$$

is affinity matrix then the minimum Ncut among $L$ and $M$ is:

$\min \operatorname{Ncut}(L, M)=\min \frac{x^{T}(P-Q) x}{x^{T} Q x}$

where $x$ is orthogonal to second lowest eigenvectors $e_{1}, e_{2}, e_{3}, \ldots, e_{n}$ of $\left[\frac{P-Q}{P}\right]$ is called as Rayleigh Quotient [20].

- If $x \in R$ then solve the generalized eigen value problem to minimize Rayleigh Quotient $(P-Q) x=\lambda x$

Normalized cut value is generated with the help of the eigen vector $e_{2}$ corresponding to second smallest eigen value.

\section{ENHANCED N-CUT AND WATERSHED BASED ALGORITHM}

Image segmentation is equivalent to the problem of partitioning the graph. To segment an image by graph theoretical approach, initially image gets converted into the graphical structure. Graphical structure of an image will have nodes which indicates pixels of an image and pixel intensities are the weights of edges among the nodes. These type of structures of an image are more flexible and computationally effective way for the design of image segmentation problem.

\subsection{Initial Segmentation}

Gradient Watershed transform is used for the primary grouping of the MRI, which generates primitive regions.

Blue Eyes Intelligence Engineering

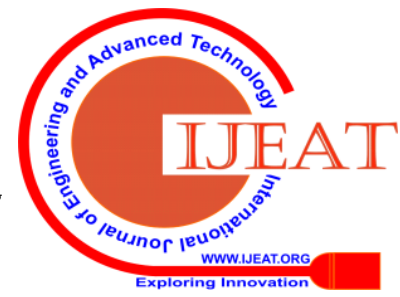


Firstly, in the gradient image of the MRI object counters will have high pixel values whereas low pixel values are scattered all over in the remaining region of an image in which local minima needs to be removed after that apply gradient watershed transform [21]. It yields consistent sections and arcs corresponding to segment borders. Actual image and partitioned image with consistent sections generated by watershed transform are as shown in fig. 1(a) and fig. 1(b) respectively.

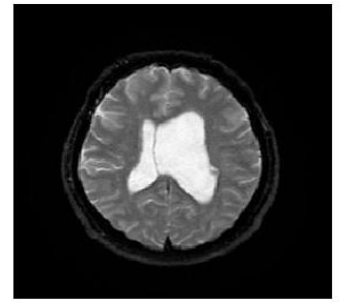

Fig. 1(a): Actual Image

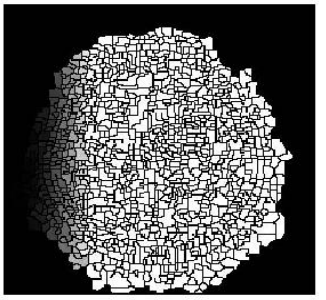

Fig. 1(b) Partitioned Image

\subsection{Watershed Enhanced Normalized Cut Algorithm (WENCA)}

Every part of output image of the first step is treated as a vertex of the graph and linking between them is represented by edges. These edges will be assigned with weight which is similarity index among the pixels. Weight of an edge connecting to the node a and $\mathrm{b}$ is determined using Eq. (7) by considering brightness and spatial location of pixels,

$l_{k s}=e^{\frac{-\|V(k)-V(s)\|_{2}^{2}}{\sigma_{F}}} * \begin{cases}e^{\frac{-\left\|L_{s}-L_{k}\right\|_{2}}{\sigma_{L}}}, & \text { if }\left\|L_{s}-L_{k}\right\|_{2}<r \\ 0, & \text { otherwise }\end{cases}$

where $L_{k}$ is the dimensional position of node a. $V(k)$ is a feature vector depending on intensity and color of node $a$.

$\sigma_{F}$ and $\sigma_{k}$ are dimensional regulating constraints respectively. $l_{k s}$ is an element of affinity matrix $Q$.

$\sigma_{F}$ is regulating constraint that monitors magnitude of the feature intensity modification included in determining $l_{u s}$. Eq. (7) indicates that if the values of $\sigma_{F}$ and $l_{k s}$ are small then

the pixels will be falling in closed groups which will lead to maximum local segmentation and vice versa.

The regulating constraint $\sigma_{L}$ monitors grade of the dimensional feature included in determining $L$. For the constant values of $\sigma_{F}$ and $\sigma_{L}$ quality of segmentation is compromised, due to constant values it generates global segmentation that ignores local changes in an image. For improving the segmentation quality, we interrelated the feature parameters about pixel $k$ and $s$ by demonstrating $\sigma_{F}$ as

$\sigma_{F}=[\sigma(V(k), a) \cdot \sigma(V(s), a)]^{-1}$

$\sigma(V(k), a)$ and $\sigma(V(s), a)$ are the S. D. of neighboring features about pixel $k$ and pixel $s$ respectively, within the radius a. $\sigma_{F}$ modelled in Eq. (8) seizes the interrelation of adjacent features among pixels $k$ and s while calculating the weights of edges. For the constant range; for reduced values of $\sigma(V(k), a)$, local dissimilarities about pixel $k$ will be lesser same will be the case for pixel s. In addition to this for small change in mutual local features about pixel $k$ and pixel $s$, the value of $\sigma(V(k), a) \cdot \sigma(V(s), a)$ will be less and hence enhanced $L_{k s}$. Proposed $\sigma_{F}$ is favorable to the fact that there should be strong weight linking among the alike adjacent pixels of the affinity matrix $Q$, which leads to better quality of segmentation with linear complexity.

Determine the eigen vector for smallest eigen value using Eq. (9)

$$
(P-Q) x=\lambda P x
$$

Change general eigen scheme to the typical eigen value problem using eq. (10).

$P^{-\left(\frac{1}{2}\right)}(P-Q) P^{-\left(\frac{1}{2}\right)} x=\lambda x$

For $k$ vertices in the graph total quantity of operations required to determine all eigen vectors are $O\left(k^{3}\right)$ which is very big number and not feasible for segmentation application. But the characteristic of local links in graphs to be partitioned helps to use the few top eigen vectors for partitioning and it reduce the computations to $O\left(k^{\frac{3}{2}}\right)$.Determine the eigen vectors, divide the graph into two portions with the help of second smallest vector. Recursively divide each part and stop the procedure when Ncut is beyond threshold value.

\section{EVALUATION AND PERFORMANCE ANALYSIS}

For the performance analysis of proposed algorithm WENCA simulations are carried out with MATLAB on a standard laptop of $5^{\text {th }}$ Gen Intel Core i7 processor; for the images from the Brain Web Simulated Brain Database [22].

Parameters chosen for enhanced segmentation quality are number of classes $C=4$ which are cerebrospinal fluid, white matter, grey matter and background, vector format for representing pixels will have pixel grey levels. We have selected three noise levels ( $0 \%, 5 \%$ and $9 \%$ ) for Simulation. Segmentation results for brain MR image by using FCM and WENCA for distinct noise levels are illustrated in figures 2.

\begin{tabular}{|c|c|c|}
\hline & \\
\hline $\begin{array}{c}\text { MR Image } \\
(0 \% \text { Noise })\end{array}$ & FCM on MR & Image (0\% Noise) \\
\hline
\end{tabular}

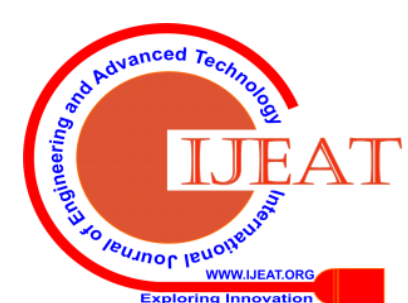


Enhanced N-Cut and Watershed based Model for Brain MRI Segmentation

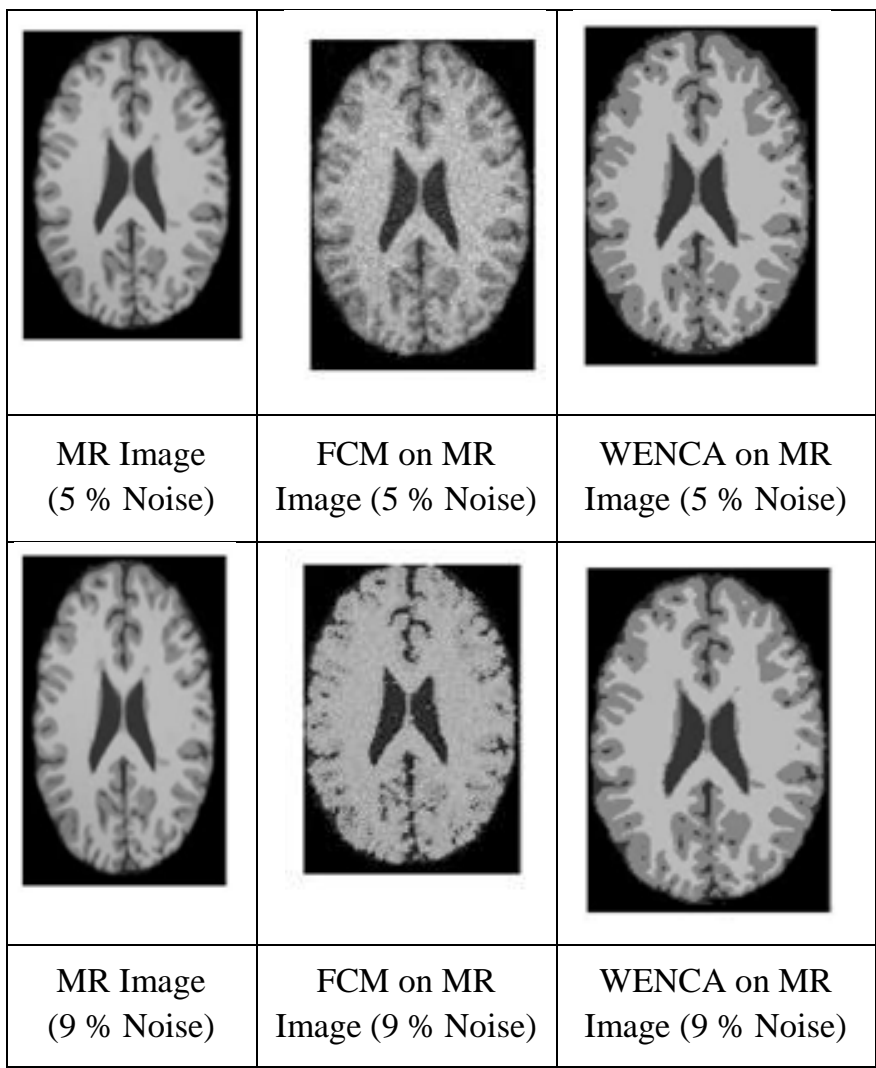

Fig. 2: Segmentation of Brain MR Image $(C=4)$

Quality of segmentation obtained by WENCA is superior than the conventional FCM, this is due to the that the parameter designed in proposed approach concentrates more on local changes in the image than the global one. With the increase in noise level, quality of segmentation by FCM degrades swiftly.

Healthy brain tissue can be divided into three categories like; White Matter (WM), Cerebro-Spinal Fluid (CSF) and Grey Matter (GM). Figure 3 illustrates results for brain tissue extraction from MR image for both the approaches with $9 \%$ level of noise.

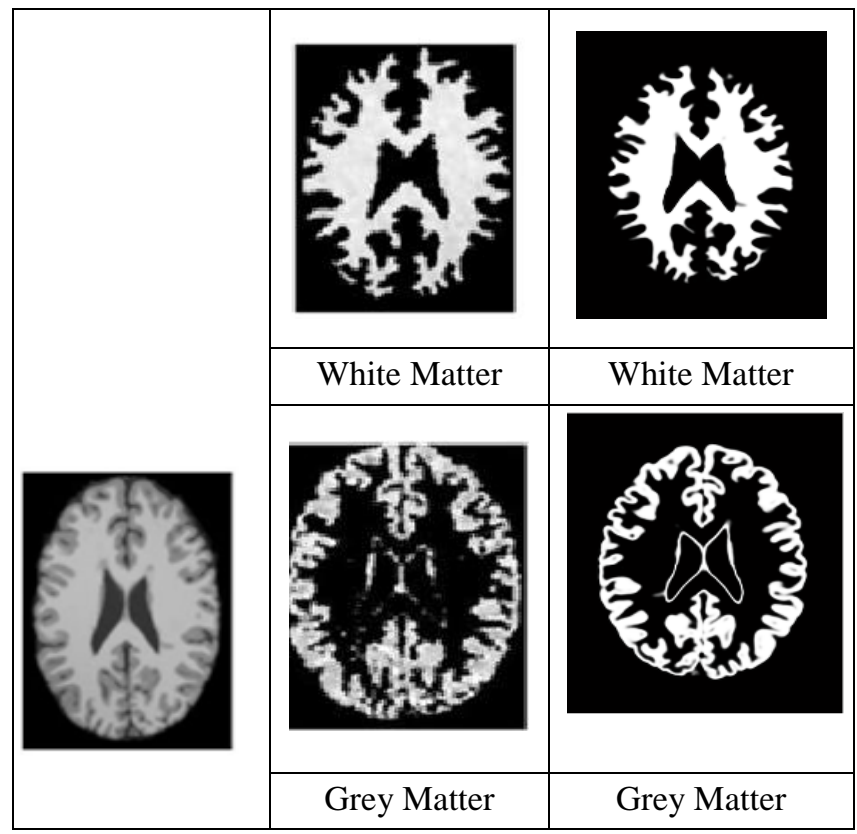

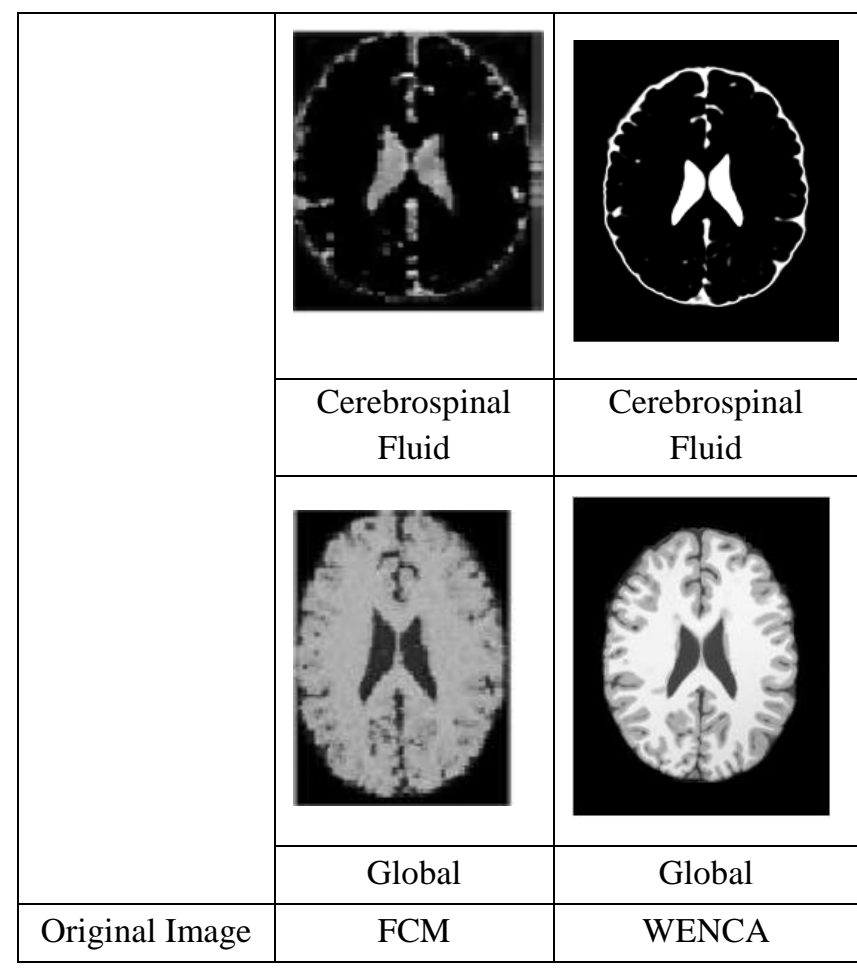

Fig. 3: Brain tissue extraction from MR Image with 9\% Noise level

Quantitative performance anlysis of the proposed approach for brain MRI segmentation is done on the basis of Probabilistic Random Index (PRI) [23] and Uniformity Error (UE) [24]. These two metirics consider the parameters common to the algorithmic segmentation and ground truth segmentation.

The proportion of the pixel pairs having identical label linking among two segment sections is the Probabilistic Random Index (PRI).

If $B_{1}, B_{2}$ are the segments with tags $b_{u}, b_{v}$ respectively for $K$ pixels $x_{1}, x_{2}, \ldots, x_{K}$ then the $\mathrm{RI}$ is

$R\left(B_{1}, B_{2}\right)=\frac{2}{K(K-1)} \sum_{u, v}\left[I\left(b_{u}=b_{v} \Lambda \mathrm{b}_{\mathrm{u}}^{\prime}=b_{v}^{\prime}\right)+\left(b_{u} \neq b_{v} \Lambda \mathrm{b}_{\mathrm{u}}^{\prime} \neq b_{v}^{\prime}\right)\right]$

$0 \leq R I \leq 1 ; 0$ signifies wide-ranging variation and 1 signifies that segments are almost alike.

PRI for the evaluation among ground truth and algorithmic segmentation is:

$\operatorname{PR}\left(O_{\text {test }},\left\{O_{g}\right\}\right)=\frac{2}{K(K-1)} \sum_{u, v} l_{u v} m_{u v}\left(1-l_{u v}\right)\left(1-m_{u v}\right)$

where $u<v, O_{\text {test }}$ is the segmentation obtained by using algorthm and the ground truth segmentation is $O_{g}, m_{u v}$ are the pixels having same labels in $O_{\text {test }}$ whereas $l_{u v}$ repesents probabilty that u and v posses idential lable $O_{g}$.

$0 \leq P R I \leq 10$ signifies wide-ranging dissimilarity and 1 signifies that $O_{\text {test }}$ and $O_{g}$ are almost identical. Homogeneity in the segmentation outcomes is evaluated by error metric. Pixel p lies in the segments $O_{1}$ and $O_{2}$ such that $O_{1} \mathrm{CO}_{2}$ with the local error is 0 then the pixel $\mathrm{p}$ will be the member of enhancement region.

Published By:

Blue Eyes Intelligence Engineering

\& Sciences Publication 
If not, then there exists overlap between the regions.

The local refinement error is:

$$
E\left(O_{1}, O_{2}, p\right)=\frac{\left|E\left(O_{1}, p\right)-E(O, p)\right|}{\left|E\left(O_{1}, p\right)\right|}
$$

$E\left(O_{1}, p\right)$ is group of pixels from the section of segment $O_{1}$ containing pixel p. Global Uniformity Error (GUE) specifies unifecial local enhancements and Local Uniformity Error (LUE) specifies multfecial enhancement in diverse portions of an image. For k amount of pixels GUE and LUE are:

$$
\begin{aligned}
& \operatorname{GUE}\left(O_{1}, 0\right)=\frac{1}{k} \min \left\{\sum_{\forall k} E\left(O_{1}, O_{2}, p\right), \sum_{\forall n} E\left(O_{2}, O_{1}, p\right)\right\} \\
& \operatorname{LUE}\left(S_{1}, S_{2}\right)=\frac{1}{k} \sum_{\forall k} \min \left\{E\left(O_{1}, O_{2}, p\right), E\left(O_{2}, O_{1}, p\right)\right\}
\end{aligned}
$$

Quality metrics are; Mean Square Error (MSE), and Peak Signal to Noise Ratio (PSNR) [25]. MSE is the alterations among the pixel intensity of an original and segmented image.

$$
M S E=\frac{1}{\operatorname{lm}} \sum_{j=1}^{l} \sum_{k=1}^{m}\left(O_{1}(r, s)-O_{2}(r, s)\right)^{2}
$$

Larege values of MSE specifies greater variations among the segmented and actual image regions. Peak Signal to Noise Ratio (PSNR) is:

$$
P S N R=\left|20 . \log _{10} \frac{255}{\sqrt{M S E}}\right|
$$

Improved quality of processed image is signified by the larger value of PSNR.

Quantitative Analysis of Brain MRI Image Segmented by FCM and WENCA is illustrated in Table 1.

WENCA generates the segmentation close to the ground truth segmentation, hence the pixels having identical labels as that

of ground truth will be higher therefore the chances of having identical label accuracy will also be more. We can observe that due to this reason PRI for the WENCA is around 0.9; which specifies enhanced segmentation outcomes.

Error metric values for WENCA are reasonably small, it indicates that WENCA generates segmentation comparable to the ground truth segmentation.

PSNR and MSE values of an image segmented by FCM and WENCA are calculated and it has been observed that WENCA produces best quality segmentation with highest PSNR and lowest MSE values.

Table 1: Quantitative Analysis of Brain MRI Image Segmented by FCM and WENCA

\begin{tabular}{|c|c|c|c|c|l|l|}
\hline \multirow{2}{*}{ Algorithm } & Noise & PRI & GUE & LUE & PSNR & MSE \\
\hline \multirow{2}{*}{ FCM } & $0 \%$ & 0.841 & 0.071 & 0.113 & 23.032 & 139 \\
\cline { 2 - 7 } & $5 \%$ & 0.813 & 0.102 & 0.161 & 20.613 & 188 \\
\cline { 2 - 7 } & $9 \%$ & 0.796 & 0.091 & 0.142 & 22.034 & 183 \\
\hline \multirow{2}{*}{ WENCA } & $0 \%$ & 0.923 & 0.050 & 0.065 & 24.661 & 114 \\
\cline { 2 - 7 } & $5 \%$ & 0.907 & 0.051 & 0.071 & 23.913 & 117 \\
\hline
\end{tabular}

\begin{tabular}{|l|l|l|l|l|l|l|}
\hline & $9 \%$ & 0.891 & 0.054 & 0.078 & 22.517 & 121 \\
\hline
\end{tabular}

\section{CONCLUSION}

In this paper we have proposed hybrid approach by combining enhanced normalise cut and watershed algorithm for segmentation of MR brain images and developed watershed enhanced normalised cut algorithm (WENCA). Prelimary partition of MRI is generated by using watershed transform to create primitive regions. Then the enhanced nomalised cut by tuning of parameters is developed, which focuses on local features of an image. In the next stage these primitive regions resembled for graph depiction and then the normalized cut method is used for segmenting an image.

We have compared the performance of WENCA with the traditional FCM method. Segmentation results obtained by proposed approach shows a noteworthy improvement in comparison with FCM for all the noise levels. Improved segmentation obtained by WENCA, lead to the acceptable extraction of the distinct tissues; GM, WM and CSF. The proposed hybrid approach will be helpful for applications in medical image segmentation.

\section{REFERENCES}

1. Chaozhe, Z.; Jiang,T,. Metacontext fuzzy clustering for separation of brain tissues in magnetic resonance images. Neuro Image. 2003.18(3), 685-696.

2. Shan S.; William S.; Malcolm G. and Annette S. MRI Fuzzy Segmentation of Brain Tissue Using Neighborhood Attraction with Neural-Network Optimization. IEEE Trans. on Info Tech in Biomed. 2005. 9(3), 459-467.

3. Wells W.; Grimson W.; Kikinis R. Adaptive segmentation of MRI data. IEEE Trans, Med. Imag. 1996. 15, 429- 442.

4. Guttmann, C.; Jolesz, F; Kikinis A; Killiany, R.; Moss, M.; Sandor, T. and Albert, M. White Matter Changes with Normal Aging. Neurology. 1997. 50, 972-978.

5. Niessen W.; Vincken K.; Weickert J.; Haar B.; Viergever M. Multiscale segmentation of 3D Brain MR Images. Internat. J. Comput Vision. 1999.31 (2/3),185-202.

6. Suzuki H; Toriwaki J. Automatic segmentation of head MRI images by knowledge guided thresholding. Computerized Medical Imaging and Graphics. 1991. 15(4), 233-240.

7. Elnakib A.; Gimel'farb G.; Suri J.; and El-Baz. A. Medical Image Segmentation: A Brief Survey, Multi-Modality State-of-the-Art Medical Image Segmentation and Registration Methodologies. Springer, 2011. 1-39.

8. Maji P.; and Paul S. Rough-Fuzzy Clustering for Grouping Functionally Similar Genes from Microarray Data. IEEE/ACM Trans. on Comp. Bio. and Bioinfo. 2018. 10(2), 286-299.

9. Koley, S.; and Majumder M. Brain MRI segmentation for tumor detection using cohesion based self-merging algorithm. Proc. of the IEEE 3rd Int. Conf on Comm. Soft. and Net. (ICCSN), Xi'an. 2017. 781 -785 .

10. Bueno G.; Musse O.; Heitz F.; Armspach J. 3D Watershed-based segmentation of internal structures within MR brain images. Medical Images 2000: Image processing, Proc. SPIE. 2000. 284-293.

11. Senthil N.; and Rajesh R. Edge Detection Techniques for Image Segmentation - A Survey of Soft Computing Approaches. International Journal of Recent Trends in Engineering. 2009. 1(2), 210-217.

Published By:

Blue Eyes Intelligence Engineering

\& Sciences Publication

(C) Copyriaht: All rights reserved. 


\section{Enhanced N-Cut and Watershed based Model for Brain MRI Segmentation}

12. Inerney T.; Terzopoulos D.; Deformable Models in Medical Image Analysis: A Survey. Medical Image Analysis. 1996. 1(2), 91-108.

13. Ji L.; and Yan H. An Attractable Snakes based Greedy Algorithm for Contour Extraction. Pattern Recognition. 2008. 35 (4), 791-806.

14. Karen Chia-R.; Miin-Shen Y.; Hsiu-Chih L.; and Pei-Ning W. Generalized Korhonen's Competitive Learning Algorithm for Ophthalmological MRI Segmentation. Magnetic Resonance Imaging. 2003. 21, 863- 870 .

15. Ozkan M.; Dawant B.; and Maciunas R. NN based Segmentation of Multimodal Medical Images: A Comparative and Prospective Study. IEEE Trans Medical Imaging. 1993. 12, 534-544.

16. De Bock J.; De SmetP.; and Philips W. Image segmentation using Watersheds and Normalized Cuts. SPIE Electronic Imaging, 2003.

17. Wu Z.; and Leahy R. An optimal graph theoretic approach to data clustering: Theory and its Application to Image Segmentation. IEEE Trans. Pat. Ana. and Mach. Intl. 1993. 15,1101-1113.

18. Shi J.; and Malik J. Normalized Cuts and Image Segmentation. IEEE Trans. Pat. Ana. and Mach. Intl. 2000. 22(8), 888-905.

19. Golub G.; and Loan C. Matrix Computations. John Hopkins Press, 1989.

20. Meyer F. Hierarchies of partitions and morphological segmentation. in Proc. of Scale-Space. 2001. 161-182.

21. Brain Web: Simulated Brain Database, McConnell Brain Imaging Centre, Montreal Neurological Institute McGill, 2014.

22. Polak M.; Zhang H.; Pi M. An evaluation metric for image segmentation of multiple objects. Image and Vision Computing. 2009. 27 (8). 1223-1227.

23. Martens J.; and Meesters L. Image dissimilarity. Signal Processing. 2008. 70, 155-176.

24. VQEG, Final report from the video quality expert group on the validation of objective models of video quality assessment, 2000.

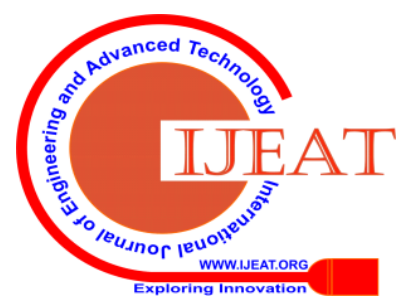

